\title{
MRI Segmentation based on Multiobjective Fuzzy Clustering
}

\author{
Olfa Mohamed Limam \\ Institut Supérieur d'Informatique, University Tunis EL Manar, Tunis, Tunisie \\ limemolfa@yahoo.fr
}

\begin{abstract}
Brain image segmentation has a major role in medical image analysis for better interpretation of complex medical diagnosis such as tumor detection. The challenge of brain tumor detection is to detect accurately the tumor portion inside the brain image. In this work, we propose a multiobjective clustering framework to separate tumor regions from a brain image based on the neighbor nearest strategy. Applied to magnetic resonance image brain, our method provides an accurate identification of brain tumor.
\end{abstract}

Keywords: Brain tumor detection, fuzzy clustering, multiobjective optimization, neighbor nearest strategy.

\section{Introduction}

Brain tumor detection is a challenging task in medical image processing [1]. Brain tumor detection problem is termed as clustering problem while it consists in partitioning a given image into different regions [3]. Therefore, it is obvious to apply clustering for the distinction of tumor tissues from other healthy tissues for medical images [4].

Most conventional clustering methods assign each pixel to a one single region. While, boundaries between regions are not clearly defined [4]. So, fuzzy clustering is more appropriate to detect tumor in brain images [5]. Fuzzy clustering has been widely applied for brain image segmentation [6]. Li et al. [7], Pham and Prince [8] used FCM algorithm. Maksoud et al. [9] used K-means clustering technique combined with FCM algorithm. Udupa and Pnuam [10] used the fuzzy connectedness for abnormal tissue segmentation. However, these methods are very sensitive to noise. Hence, hybrid methods was applied in order to get desired results. Menon et al. [11] and Alsmadi [12] combined FCM with artificial bee colony algorithm. However, single fuzzy clustering is not recommended since single validity index fails to cope with different types of data sets. Moreover, the wrong choice of a single clustering measure may conduct to unsatisfying segmentation results [5]. Then, several multiobjective approaches have been proposed to segment brain images [13]. Acharya et al. [14] used simulated annealing for classification of cancer data sets. Three cluster validity indices are optimized namely XB, PBM, and FCM indices, to accurately reflect tissue clusters. Mukhopadhyay et al. [15] used NSGA-II to optimize the same three objective functions. Saha and Bandyopadhyay [16] proposed a genetic clustering technique and in [17] they proposed variable length genetic clustering technique to segment brain image data sets. 
However, most multiobjective fuzzy clustering techniques are developed for brain image segmentation and not for tumor detection and optimized with at most two objective functions. In previous work, Limam [18] proposed a multiobjective fuzzy genetic clustering technique optimizing two objective functions, the spatial compactness and the spatial separateness of clusters for brain tumor detection.

In this work, we propose a multiobjective fuzzy clustering method for brain tumor detection using three objective functions based on different data properties, namely, the fuzzy neighbor nearest connectedness, the fuzzy variance cluster and the external c cluster validity index Minkowski score. Our method generates an ensemble of Pareto solutions and we use the Minkowski score to select the final segmented image. Therefore, this paper develops a new multiobjective fuzzy clustering approach for brain tumor detection in MRI images to accurately diagnose the region of cancer. The main problem of works is that the quantitative results done by different works for their proposed methods are tested on different datasets rather than a common standard dataset. Also, the absence of a standard measure to compare classification accuracy of algorithms .

This paper is organized as follows. Section 2 details our proposed method. Section 3 illustrates the experimental study. Section 4 presents a conclusion.

\section{The Multiobjective Fuzzy Clustering Method}

The different steps of our proposed method are detailed in the following sections.

\subsection{Color features}

In order to improve the quality of the resulting segmentation, color features are used [19]. The standard RGB color space is used by our proposed algorithm.

\subsection{Pattern proximity}

The Euclidean distance is used to calculate the pattern proximity [20]. In general, the distance between two pixels $x:\left(x_{1}, \ldots, x_{n}\right)$ and $y:\left(y_{1}, \ldots, y_{n}\right)$ in an Euclidean $n$-space is given by

$$
d(x, y)=|x-y|=\sqrt{\sum_{i=1}^{n}\left|x_{i}-y_{i}\right|^{2}}
$$

\subsection{Multiobjective fuzzy clustering algorithm}

NSGA-II is adopted as the underlying multiobjective framework for fuzzy clustering. NSGA-II inputs arguments are the population size, an upper bound of the number of clusters, the data set, and a maximum number of generations. At the beginning of the algorithm, an initial potential solutions should be defined.

\subsubsection{Initialization Step}

In NSGA-II based clustering, chromosomes encodes the centers of the partitions. So, in the initial NSGA population, initial centers are encoded using FCM in order to provide more accurate solutions. FCM produces $\mathrm{C}$ cluster centers and a $\mathrm{C} \times \mathrm{N}$ membership matrix $\mathrm{U}(\mathrm{x})$. 


\subsubsection{Fitness Functions Computation}

Three cluster validity measure are used to quantify the quality of each obtained chromosome. The fuzzy neighbor nearest connectedness index, fuzzy variance of clusters and Minkowski index, are simultaneously optimized and computed for each chromosome. To compute the objective functions, we extract the centers encoded in a given chromosome. The fuzzy neighbor nearest connectedness $\mathrm{NN}_{\mathrm{c}}$ index, is defined by

$$
N N_{c}=\sum_{i=1}^{C} \frac{\sigma_{i}}{n_{i}}=\sum_{i=1}^{C} \frac{\sum_{k=1}^{N} f_{i k}^{m} D\left(v_{i}, x_{k}\right)}{\sum_{k=1}^{N} f_{i k}^{m}}
$$

where $m$ the fuzzy exponent, $D\left(v_{i}, x_{k}\right)$ defines the Euclidean distance between $i^{\text {th }}$ cluster center and $k^{\text {th }}$ data point, $\sigma_{i}$ denotes the variation of clusters and $n_{i}$ the fuzzy cardinality of the $i^{\text {th }}$ cluster is given by:

$$
n_{i}=\sum_{k=1}^{N} f_{i k}^{m}, 1 \leq i \leq C
$$

The conditional membership function of a pixel $f_{i k}$ is defined as

$$
f_{i k}=\frac{\boldsymbol{u}_{i k} \boldsymbol{h}_{i k}}{\sum_{j=1}^{C} \boldsymbol{u}_{j k} \boldsymbol{h}_{j k}}
$$

where the membership degree $u_{i k}$ is defined as

$$
u_{i k}=\frac{1}{\sum_{j=1}^{C}\left(\frac{D\left(v_{i}, x_{k}\right)}{D\left(v_{j}, x_{k}\right)}\right)^{\frac{2}{m-1}}}
$$

and $h_{i k}$, the level of pixel $x_{k}$ belonging to the $i^{\text {th }}$ cluster based on its neighborhood in a spatial domain, is defined as follows:

$$
h_{i k}=\sum_{k \in N b\left(x_{k}\right)} g_{i k}
$$

with

$$
g_{i k}=\left\{\begin{array}{l}
1 \text { if } u_{i k}=\max \left\{u_{l k}\right\}, \text { for } \quad l=1, \ldots, C \\
0 \quad \text { otherwise }
\end{array}\right\}
$$

where $\mathrm{NB}\left(\mathrm{x}_{\mathrm{k}}\right)$ represents a square neighborhood having pixel $\mathrm{x}_{\mathrm{k}}$ in its center. We used a pixel Window of $3 \times 3$. The fuzzy variance of clusters $V$ is given by 


$$
V=\sum_{i=1}^{C} \sum_{i=1, j \neq i}^{C} f_{i j}{ }^{m} D\left(v_{i}, v_{j}\right) .
$$

where $D\left(v_{i}, v_{j}\right)$ the Euclidean distance between clusters $v_{i}$ and $v_{j}$.

The external cluster validity index Minkowksi score (MS), measuring the agreement between the true clustering $T$ and the obtained clustering $U$, is given by:

$$
M S(T, U)=\sqrt{\frac{n_{01}+n_{10}}{n_{11}+n_{10}}}
$$

where $\mathrm{n}_{11}$ is the total number points assigned to the same clusters in both $T$ and $U, n_{01}$ the total number of pairs of points that are assigned only in the same cluster of $U$ and $n_{10}$ the total number of pairs of points that are assigned to the same cluster of $T$ but in a different cluster of U. Hence, the fuzzy neighbor nearest connectedness measure $\mathrm{NN}_{\mathrm{c}}$ should be minimized, the fuzzy variance $\mathrm{V}$ should be maximized and the Minkowski score MS should be minimized, as follows:

$$
\left\{\begin{array}{l}
\text { Min NN } \\
\text { Max V } \\
\text { Min MS }
\end{array}\right.
$$

\subsubsection{Genetic Operators}

Crowded binary tournament selection is adopted to generate the mating set of chromosomes, conventional crossover and mutation are utilized. The NSGA-II final step is its elitism operation, where the Pareto solutions among the parent and child populations are propagated to the next generation. The NSGA based clustering algorithm provides a set of solutions on the final Pareto optimal front.

\subsection{Optimal solution}

The final part of the multiobjective fuzzy clustering algorithm is chosen as the best solution from a set of solutions based on a validity measure MS index [21]. The best partition corresponds to the minimum value of MS index [21].

\section{Experiments and Analysis}

Segmentation results are analyzed based on a visual experimental study applied to three simulated brain tumor image data sets illustrating different tumor locations. The following Figures present different tumor types inside the brain stem. The simulated brain MR images can be downloaded from Brainweb [22].

The evolutionary parameters of our method are set as follows: the population size is 20 , the number of generations is equal to 20 , the crossover probability is 0.8 and mutation probability is set to be 0.01 . The number of clusters used are between 2 and 16 and the fuzzy factor $m$ is set to 2 . The (a) parts of Figures 
Olfa Mohamed Limam; MRI Segmentation based on Multiobjective Fuzzy Clustering. Journal of Biomedical Engineering and Medical Imaging, Volume 3, No 2, April (2016), pp 7-13

(1), (2) and (3) show the original MRI brain tumor images and the (b) parts show the segmented images generated by our method.
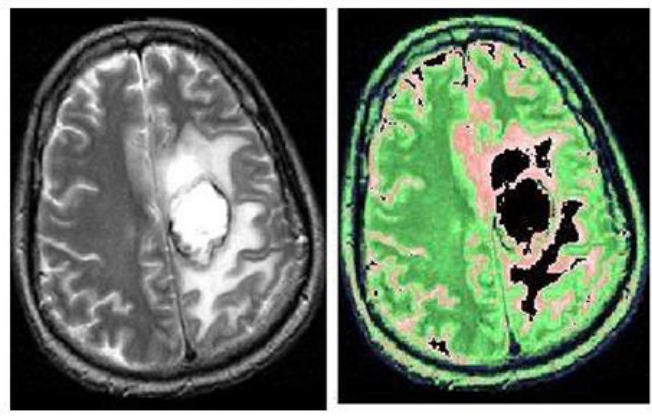

Figure 1 (a) Original MRI brain tumor image (b) Image segmented by our method
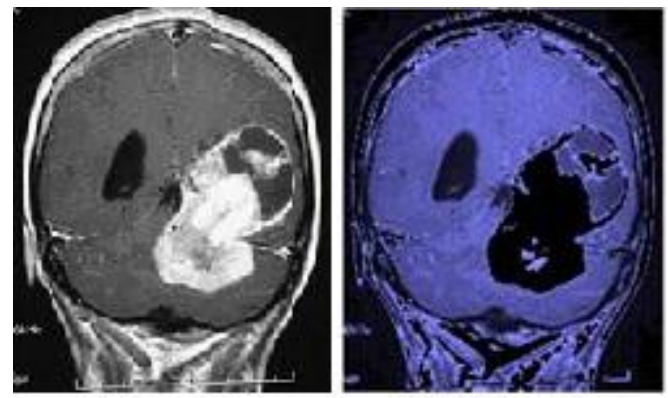

Figure 2 (a) Original MRI brain tumor image (b) Image segmented by our method
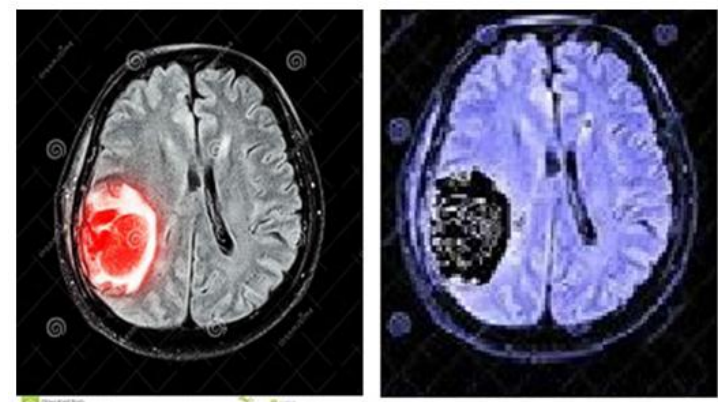

Figure 3 (a) Original MRI brain tumor image (b) Image segmented by our method

In previous Figures, our method succeeds to locate the tumor part of the brain and clearly separates

it from the other parts of the brain. Results shows that for the different images, our method makes a clear identification of the tumor portion in the brain.

\section{Conclusion}

This paper presents a multiobjective fuzzy clustering method that optimizes three objectives. A visual comparison applied to MRI brain image was conducted in order to show the effectiveness of our proposed method to detect brain tumor images. Our proposed approach can be extended to detect other types of tumors in other medical imagery types. 


\section{REFERENCES}

[1] Shen Shan, Sandham William, Granat Malcolm and Sterr Annette. Mri fuzzy segmentation of brain tissue using neighborhood attraction with neural-network optimization. IEEE Transactions on Information Technology in Biomedicine, 9(3):459-467, 2005.

[2] P.Tamije Selvy, V. Palanisamy, and T. Purusothaman. Performance analysis of clustering algorithms in brain tumor detection of $\mathrm{mr}$ images. European Journal of Scientific Research, 62(3):321-330, 2011.

[3] Suchita Yadav and Meshram Sachin. Brain tumor detection using clustering method. International Journal of Computational Engineering Research, 3(4):11-14, 2013.

[4] Miin-Shen Yang, Yu-Jen Hu, Karen Chia-Ren and Charles Chia-Lee Lin. Segmentation techniques for tissue differentiation in mri of ophthalmology using fuzzy clustering algorithms. Magentic resonance Imaging, 20:173-179, 2002.

[5] Sanghamitra Bandyopadhyay, Anirban Mukhopadyay, and Ujjwal. Maulik. Combining multiobjective fuzzy clustering and probabilistic ANN classifier for unsupervised pattern classification: Application to satellite image segmentation. IEEE on Evolutionary Computation, pages 877-883, 2008.

[6] Weibei Dou, Su Ruan, Daniel Bloyet, and Jean-Marc Constans. A framework of fuzzy information fusion for the segmentation of brain tumor tissues on $\mathrm{mr}$ images. Image and Vision Computing, 25(2):164-171, 2007.

[7] Chunlin Li, DB Goldgof, and LO Hall. Knowledge-based classification and tissue labeling of $\mathrm{mr}$ images of human brain. IEEE transactions on Medical Imaging, 12(4):740-750, 2002.

[8] L. Dzung Pham and Jerry L. Prince. Knowledge-based classification and tissue labeling of $\mathrm{mr}$ images of human brain. IEEE transactions on Medical Imaging, 18(9):737-752, 1999.

[9] Eman A. Abdel Maksoud, Mohammed Elmogy, and Rashid Mokhtar Al-Awadi. MRI brain tumor segmentation system based on hybrid clustering techniques. In the Second International Conference of Advanced Machine Learning Technologies and Applications -, AMLTA, pages 401412, 2014.

[10] Jayram K. Udupa and K. Saha Punam. Fuzzy connectedness and image segmentation. Proceedings of the IEEE, 91:1649-1669, 2003.

[11] N. Menon, R. Ramakrishnan. Brain tumor segmentation in mri image using unsupervised artificial bee colony and $\mathrm{fcm}$ clustering. International Journal of Computer Science and Management Research, 2:2450-2454, 2013.

[12] Mutasem K. Alsmadi. Mri brain segmentation using a hybrid artificial bee colony algorithm with fuzzy-c mean algorithm. Journal of Applied Sciences, 15:100-109, 2015. 
Olfa Mohamed Limam; MRI Segmentation based on Multiobjective Fuzzy Clustering. Journal of Biomedical Engineering and Medical Imaging, Volume 3, No 2, April (2016), pp 7-13

[13] D.M. Joshi, N. K. Rana, and V.M. Misra. Classification of brain cancer using artificial neural network. International Conference on Electronic Computer Technology (ICECT), pages 112-116, 2010.

[14] Sudipta Acharya, Yamini Thadisina, and Sriparna Saha. Multi-objective clustering of tissue samples for cancer diagnosis. International Conference on Advances in Computing, Communications and Informatics, pages 1059-1064, 2014.

[15] Anirban Mukhopadhyay, Ujjwal Maulik, and Sanghamitra Bandyopadhyay. Multiobjective genetic clustering with ensemble among pareto front solutions: Application to mri brain image segmentation. In Proceedings of the Seventh International Conference on Advances in Pattern Recognition, ICAPR '09, pages 236-239, 2009.

[16] Saha Sriparna and Bandyopadhyay Sanghamitra. Mri brain image segmentation by fuzzy symmetry based genetic clustering technique. In Proceedings of the IEEE Congress on Evolutionary Computation, CEC'2007, pages 4417-4424, 2007.

[17] Saha Sriparna and Bandyopadhyay Sanghamitra. Mr brain image segmentation using a multiseed based automatic clustering technique. Fundamenta Informaticae, 97(1):199-214, 2009.

[18] limam Olfa. Brain tumor segmentation using multiobjective fuzzy clustering. Transactions on Machine Learning and Artificial Intelligence, 4(1):58-67, 2016.

[19] Bong Chin-Wei and Mandava Rajeswari. Multiobjective optimization approaches in image segmentation - the directions and challenges. International Journal of Advances in Soft Computing and Its Applications, 2(2):41-64, 2010.

[20] Anil Kumar Jain, M Narasimha Murty, and Patrick Joseph Flynn. Data clustering: A review. ACM Computing Surveys, 31(3):265-323, 1999.

[21] Abhay Kumar Alok, Asif Ekbal, and Sriparna Saha. Brain image segmentation using semisupervised clustering. Expert Systems with Applications, 52:50-63, 2016.

[22] Brainweb: Simulated brain database, 2011, available at http://www.bic.mni.mcgill.ca/brainweb. 